\title{
Pile-ups in thin foils: application to transmission electron microscopy analysis of short-range-order
}

\author{
G. SAADA $\dagger$, J. DOUIN \\ Laboratoire d'Etude des Microstructures, Unité Mixte de Recherche \\ CNRS-ONERA, 29 avenue de la Division Leclerc, BP 72, \\ 92322 Châtillon Cedex, France \\ F. Pettinari-Sturmel, A. Coujou and N. Clément \\ Centre d'Elaboration des Matériaux et d'Etudes Structurales, 29 rue Jeanne \\ Marvig, BP 4347, 31055 Toulouse Cedex, France
}

[Received 18 July 2003 and accepted in revised form 11 November 2003]

\begin{abstract}
The chemical force acting on dislocations in a short-range-ordered concentrated Ni-based alloy is investigated by post mortem and in-situ transmission electron microscopy. For this purpose, the positions of the dislocations in a pile-up are calculated, taking into account the stress relaxation at the free surfaces. The calculation shows that the distribution of the first dislocations of the pileup is only slightly affected by the presence of the free surfaces, while the length of the pile-up strongly depends on the thickness of the foil. Analysis of pile-ups in two short-range-ordered Ni-based alloys shows that the chemical force resulting from short-range order (SRO) is noticeable up to the sixth dislocation of the pileup. These results indicate the presence of very small short-range-ordered clusters, rather than a homogeneously distributed SRO.
\end{abstract}

\section{$\S 1$. INTRODUCTION}

In-situ and post mortem transmission electron microscopy (TEM) observations have demonstrated that deformation may proceed by the motion of planar arrays of dislocations, resulting in slip bands or pile-ups of dislocations (Clément 1984, Olfe and Neuhäuser 1988, Gerold and Karnthaler 1989). This is the case when the movement of dislocations in a given plane is enhanced by the movement in the same plane of previous dislocations.

In the $\gamma$ phase of concentrated Ni-based alloys, it is now well established that this behaviour is a consequence of the existence of short-range order (SRO) of ( $\left.1 \frac{1}{2} 0\right)$ type (Clément et al. 1996, Pettinari et al. 2001, Prem et al. 2002). Indeed the glide of a dislocation $\mathrm{D}_{0}$ on a given plane $\mathrm{G}$ of a short-range-ordered alloy destroys, at least partially, the SRO, which needs an energy $\gamma_{0}$ per unit surface swept by the dislocation and corresponds to a frictional force $\gamma_{0}$ per unit length opposing the

\footnotetext{
$\dagger$ Author for correspondence. Email: saada@onera.fr. 
motion of $\mathrm{D}_{0}$. If the $\mathrm{SRO}$ is completely destroyed in the area $A$ swept by $\mathrm{D}_{0}$, any dislocation gliding in $A$ moves in a disordered solid solution and experiences no SRO frictional stress, which makes its glide easier than anywhere else in the crystal. A dislocation source activated in $A$ generates dislocations which pile up against the dislocation $\mathrm{D}_{0}$ until the force on it reaches $\gamma_{0}$. The deformation proceeds therefore by the motion of pile-ups. In the case of the $\gamma$ phase of concentrated Ni-based alloys, post mortem as well as in-situ TEM observations of the alloys reveal further that the first two dislocations of the pile up are paired (Jouiad et al. 1999, Pettinari 1999, Pettinari et al. 2001), which indicates that the frictional force $\gamma_{1}$ exerted on the second dislocation is smaller than $\gamma_{0}$ (see $\S 3$ ).

Precise comparisons between the TEM observations and the estimated positions of the dislocations resulting from calculating the elastic interaction between dislocations in an infinite medium have been made (Jouiad et al. 1998, 1999, Pettinari 1999) without taking into account the screening of the effect of elastic stresses of the dislocations by the free surfaces of the thin foil. In what follows, we shall first analyse the properties of pile-ups in short-range-ordered alloys $(\$ 2)$, then evaluate the effect of elastic screening by analysing in detail the properties of screw dislocations pile-ups in thin foils $(\S 3)$ and finally $(\S 4)$ apply the results in the case of $\gamma$-phase alloys with nominal compositions (in at.\%) 65.6 Ni-26.21 Cr-2.02 Mo-1.98 W-4.19 Re and 65.88 Ni-26.07 Cr-2.03 Mo-1.98 W-4.04 Ru, respectively, and denoted $\gamma_{\text {MCRe }}$ and $\gamma_{\text {MCRu }}$, presenting evidence of SRO (Pettinari-Sturmel et al. 2002, Prem et al. 2002).

\section{§2. General Properties of PILE-UPS}

\subsection{General pile-up in disordered alloys}

We analyse the equilibrium of an array of $n+1$ parallel dislocations of Burgers vector $\mathbf{b}$ moving in the same plane under the effect of a homogeneous applied stress $-\tau_{\mathrm{a}}$ (figure 1). Since all dislocations undergo the same applied stress $-\tau_{\mathrm{a}}$, the total force $F_{p}$ on the dislocation $p$ is:

$$
F_{p}=-b \tau_{\mathrm{a}}+S_{p}+R_{p}, \quad 0 \leqslant p \leqslant n
$$

$R_{p}$ is the total elastic force exerted on the dislocation $p$ by the other dislocations and $S_{p}$ the frictional force that each dislocation may experience owing to lattice friction and/or local interaction with foreign atoms. If $F_{i j}$ is the elastic force exerted by the dislocation $i$ on the dislocation $j$,

$$
R_{p}=\sum_{i \neq p} F_{i p}
$$

and, since one has very generally that

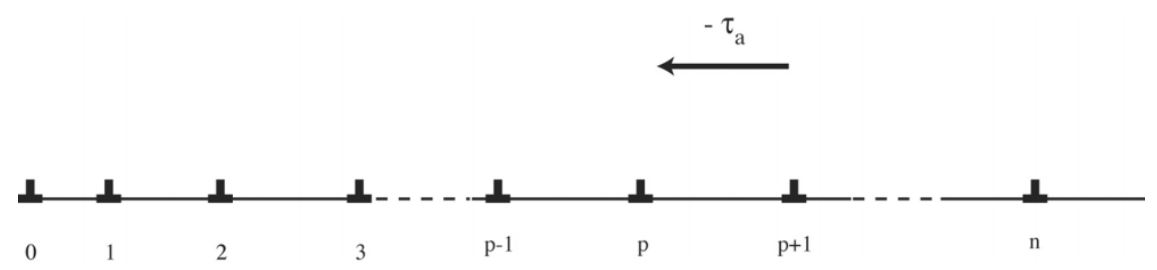

Figure 1. Schematic diagram of a general pile-up in a disordered alloy under the effect of homogeneous applied stress $-\tau_{\mathrm{a}}$. 


$$
F_{i j}+F_{j i}=0
$$

then, from equation (3),

$$
\sum_{p=0}^{p=n} R_{p}=0
$$

Whenever the frictional forces $S_{p}$ are the same for all dislocations and oppose their motion, we set

$$
S_{p}=b \tau_{\mathrm{f}}, \quad \tau_{\mathrm{f}}>0 .
$$

Then $\tau_{\mathrm{a}}$ must be replaced by $\tau_{\mathrm{a}}-\tau_{\mathrm{f}}$ in equation (1). The total force acting on the pile-up is obtained from equations (1), (4) and (5):

$$
\sum_{p=0}^{p=n} F_{p}=-(n+1) b\left(\tau_{\mathrm{a}}-\tau_{\mathrm{f}}\right) .
$$

It must be emphasized that equations (1)-(6) do not rely on any particular expression of the elastic interaction between dislocations. They are valid in an infinite as well as in a finite elastic medium.

\subsection{Pile-ups in short-range-ordered alloys}

One dislocation $\mathrm{D}_{0}$ gliding in a short-range-ordered alloy introduces some disorder. The created planar defect was defined as a diffuse antiphase boundary (DAPB) by Schwander et al. (1992). Whenever the order is completely destroyed by the glide of a single dislocation, the energy necessary to create this defect is $\gamma_{0}$. In this case, dislocations following $\mathrm{D}_{0}$ feel no frictional force resulting from the destruction of order. As a consequence, plastic flow occurs as groups of piled-up dislocations.

However, there is no reason to assume that local order is completely destroyed by the glide of the first dislocation. The SRO should change after the passage of each dislocation, and successive dislocations trail a different fault (figure 2). Assuming that, after the passage of a sufficient number of dislocations $p^{*}$, the local order is not affected any longer by the passage of other dislocations, the frictional stress $\left(\gamma_{p}-\gamma_{p-1}\right) / b$ resulting from the SRO becomes negligible for $p>p^{*}$ (since $\gamma_{p} \approx \gamma_{p-1}$ ) and thus plastic flow will occur as groups of piled-up dislocations.

Equation (1) for the total force on the dislocations is now written:

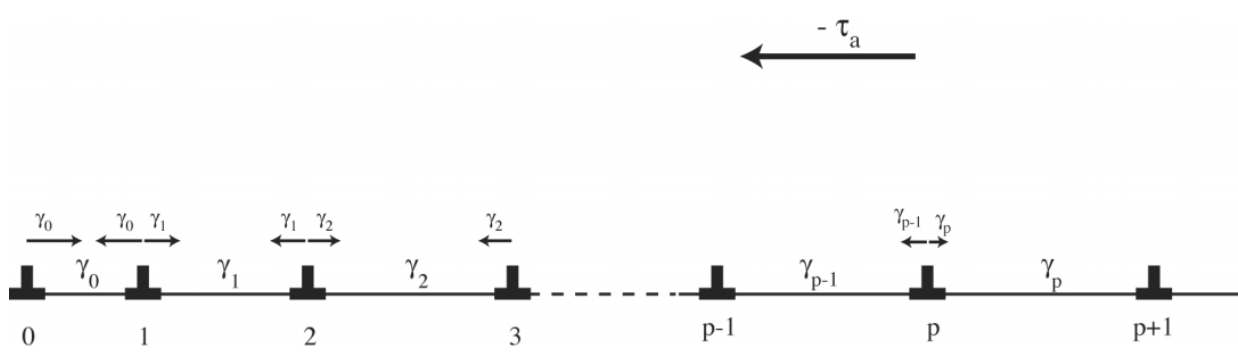

Figure 2. Schematic diagram of a pile-up in a short-range-ordered alloy under the effect of homogeneous applied stress $-\tau_{\mathrm{a}}$. 


$$
\begin{aligned}
& F_{0}=-b \tau_{\mathrm{a}}+\gamma_{0}+S_{0}+R_{0}, \\
& F_{p}=-b \tau_{\mathrm{a}}+\gamma_{p}-\gamma_{p-1}+S_{p}+R_{p}, \quad 0<p \leqslant n .
\end{aligned}
$$

Again we assume that the frictional forces $S_{p}$ are the same for all dislocations and that these oppose their motion:

$$
S_{p}=b \tau_{\mathrm{f}}, \quad \tau_{\mathrm{f}}>0 .
$$

The critical stress $\tau_{\text {eq }}$ necessary to propagate the pile-up is then obtained when the pile-up is at equilibrium:

$$
F_{p}=0, \quad 0 \leqslant p \leqslant n,
$$

which leads to the following relations:

$$
\begin{gathered}
\gamma_{0}=b\left(\tau_{\mathrm{eq}}-\tau_{\mathrm{f}}\right)-R_{0}, \\
\gamma_{p}=\gamma_{p-1}+b\left(\tau_{\mathrm{eq}}-\tau_{\mathrm{f}}\right)-R_{p}, \quad 0<p \leqslant n,
\end{gathered}
$$

or

$$
\gamma_{p}=(p+1) b\left(\tau_{\mathrm{eq}}-\tau_{\mathrm{f}}\right)-\sum_{i=0}^{p} R_{i}, \quad 0<p \leqslant n .
$$

Using equation (4), the fault energy $\gamma_{n}$ behind the pile-up is simply given by

$$
\gamma_{n}=(n+1) b\left(\tau_{\mathrm{eq}}-\tau_{\mathrm{f}}\right)
$$

where $\gamma_{n}$ is the energy resulting from the complete (or best) destruction of the SRO. This equation is independent of the expression for the force between dislocations. It is thus also valid whatever the dislocations' character, in an infinite medium as well as in a thin film. From a general point of view, equation (11) indicates that the pile-up behaves like a single dislocation with a Burgers vector $(n+1) b$ with a fault $\gamma_{n}$ in its trail.

If we assume that the local order is completely destroyed after the passage of the first dislocation, we find that

$$
\gamma_{0}=(n+1) b\left(\tau_{\mathrm{eq}}-\tau_{\mathrm{f}}\right)
$$

This case is similar to that of a pile-up of elastically interacting dislocations against an obstacle under the application of homogeneous applied stress, $-\tau_{\text {eq }}$.

Valuable information on the effect of SRO on dislocation motion as well as on the value of the DAPB energies can then be obtained, as a function of the applied stress, either by measuring the positions of the dislocations in immobile pile-ups or from the observation of the dynamic properties of metastable pile-ups. No such measurements are available in bulk materials. Post mortem and in-situ TEM observations have revealed the existence of dislocations pile-ups in short-rangeordered alloys. However, in order to obtain quantitative information, one must evaluate accurately the applied stress, and the stress between dislocations.

Direct measurement of the applied resolved shear stress in a thin film is not an easy task. All that can be done is to make reasonable assumptions and to compare them critically with the observed results. Numerical calculation of the structure of a pile-up in a thin film is, in principle, possible in any case. However, more insight is gained by a detailed analysis of simple situations and extending cautiously the results to more realistic situations. More information should be obtained from the 
in-situ TEM observation of the behaviour of pile-ups in short-range-ordered alloys, provided that the calculation of the elastic interaction between dislocations takes into account the screening of the stress field of the dislocations by the free surface of the thin foils.

\section{§3. PILE-UP IN ThIN FOILS}

\subsection{General formulation}

We now consider a thin foil of thickness $h$, containing one pile-up made of screw dislocations, either perpendicular to the surface (figure $3(a)$ ), referred to as a perpendicular pile-up, or parallel (figure $3(b)$ ) to the surface, referred to as a parallel pile-up. The dislocations are submitted to an applied stress $-\tau_{\mathrm{a}}$. The detailed calculations are developed in appendix A, $\S$ A 1 and A2. The results can be conveniently expressed in terms of the dimensionless variables $u_{i}$ and $q_{p}$ :

$$
u_{i}=\frac{x_{i}}{h}
$$

(a)

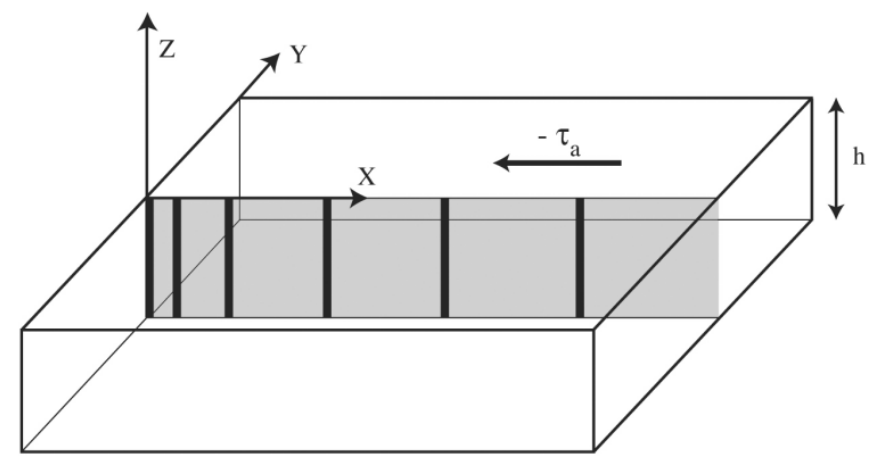

(b)

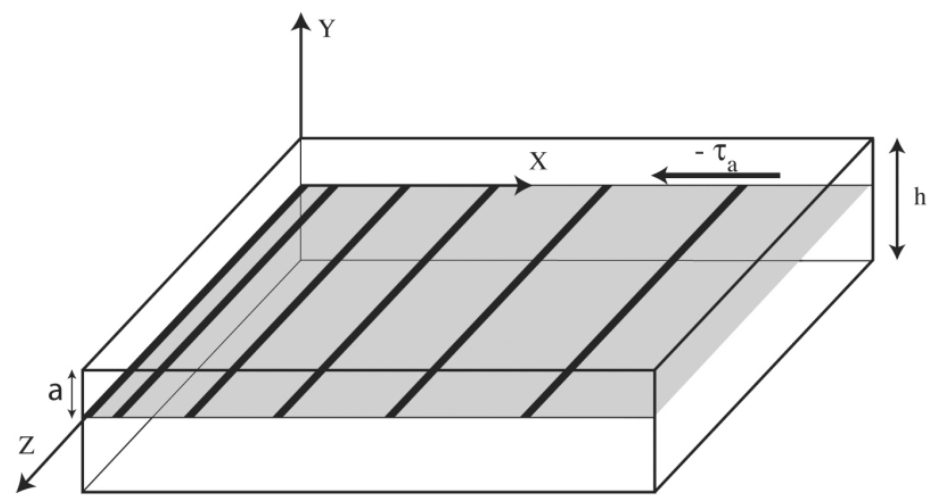

Figure 3. The two configurations in a thin foil under consideration: $(a)$ 'perpendicular' pile-up; (b) 'parallel' pile-up at a distance $a$ from the surface. 
where $x_{i}$ defines the position of dislocation $i$;

$$
\begin{gathered}
q_{0}=\frac{h}{\mu b}\left(\tau_{\mathrm{a}}-\tau_{\mathrm{f}}-\frac{\gamma_{0}}{b}\right), \\
q_{p}=\frac{h}{\mu b}\left(\tau_{\mathrm{a}}-\tau_{\mathrm{f}}-\frac{\gamma_{p}-\gamma_{p-1}}{b}\right), \quad 0 \leqslant p \leqslant p^{*}, \\
q_{p}=\frac{h}{\mu b}\left(\tau_{\mathrm{a}}-\tau_{\mathrm{f}}\right), \quad p^{*} \leqslant p \leqslant n,
\end{gathered}
$$

where $h$ is the thickness of the thin foil. With this notation, the total elastic force exerted on dislocation $j$ by the other dislocations of the pile-up has the form

$$
F_{j}=\frac{\mu b^{2}}{h}\left(f_{j}-q_{j}\right) \text {. }
$$

$f_{j}$ must be identified with $\Phi_{j}$ (equation (A 2)) for perpendicular pile-ups and with $\psi_{j}$ (equation $(\mathrm{A} 7 a)$ ) for parallel pile-ups. The conditions of equilibrium are then given by equations (9).

The positions of the dislocations at equilibrium have been calculated numerically, assuming no SRO and no frictional forces, for parallel and perpendicular pile-ups. In this case,

$$
q_{j}=q=\frac{h \tau_{\mathrm{a}}}{\mu b} .
$$

Dislocations were given arbitrary initial positions (multiples of $10 \mathrm{~nm}$ for example). They were displaced over a distance proportional to the stress that they experience and then iterated. Convergence towards the energy minimum of the total configuration was checked, and the iteration was stopped when the maximum distance run by the dislocations during a step is lower than $0.02 b$. The accuracy of the calculation procedure has been checked in four different ways:

(i) Using the final configuration as the initial configuration and increasing the precision of the iterations, we find a relative variation of the positions of the order of $10^{-3}$.

(ii) Given $n$, the positions $x_{i} / h$ (or $x_{i} / h^{*}$ ) of the dislocations depend only on $q$ (or $q^{*}$ ), for values of $\tau_{\mathrm{a}}$ and $h$ varying separately by an order of magnitude.

(iii) The calculated stress on the leading dislocation is $(n+1) \tau_{\mathrm{a}}$ within a relative error of about $10^{-3}$.

(iv) Applying the numerical procedure to the case of a pile-up in an infinite medium gives the exact values, within about $10^{-3}$, calculated either by the polynomial method (Eshelby et al. 1951), or by independent numerical methods (Chou et al. 1960).

\subsection{Perpendicular pile-up}

For a perpendicular pile-up (figure $3(a)$ ), we found that the distance $d$ between the first two dislocations of the pile-up and its total length $L$ are well represented by the following formulae:

$$
\frac{h}{d}=a_{n} q+b_{n}
$$


Table 1. Coefficients $a_{n}, b_{n}, c_{n}, d_{n}$ and $g_{n}$ as functions of $n$.

\begin{tabular}{lcccccc}
\hline & $n$ & $a_{n}$ & $b_{n}$ & $c_{n}$ & $d_{n}$ & $g_{n}$ \\
\hline Perpendicular & 6 & 21.3 & 2.2 & 0.95 & 0.75 & 1.65 \\
Perpendicular & 11 & 37.5 & 0.7 & 0.46 & 0.52 & 0.95 \\
Perpendicular & 21 & 74.6 & 2.7 & 0.25 & 0.35 & 0.6 \\
Parallel & 11 & 37.5 & 0.7 & 0.38 & 0.34 & 0.7 \\
\hline
\end{tabular}

$$
\begin{array}{ll}
\frac{h}{L}=c_{n} q+d_{n}, & 1 \leqslant q \leqslant 10, \\
\frac{h}{L}=g_{n} q^{1 / 2}, & 0.1 \leqslant q \leqslant 1 .
\end{array}
$$

The values of the coefficients $a_{n}, b_{n}, c_{n}, d_{n}$ and $g_{n}$ are given in table 1 for perpendicular pile-ups containing 6, 11 or 21 screw dislocations. Figure 4 represents the results. Note that equations (17) and (18) may be used to evaluate the stress when the total length of the pile-up is known.

\subsection{Parallel pile-up}

For a pile-up of screw dislocations in a plane parallel to the surface, the interaction forces depend on the position of the plane with respect to the surface. Let $a$ be the distance of the plane to the closest surface (figure $3(b)$ ). We show in appendix A, § A 2, that, owing to the image force, the force between two parallel screw dislocations, in a plane $\mathrm{P}$ parallel to the surface of the foil and at a distance $a$ from the closest surface, is to a very good approximation that calculated for screw dislocations at the centre of a foil of thickness $2 a$. Therefore, for a pile-up at a distance $a$ from the closest surface, we define

$$
\begin{gathered}
\alpha=\frac{a}{h}, \quad 0 \leqslant \alpha \leqslant 0.5, \\
h^{*}=2 a, \\
q^{*}=\frac{h^{*} \tau_{\mathrm{a}}}{\mu b} .
\end{gathered}
$$

Let $L_{\alpha}$ and $d_{\alpha}$ define the total length of the pile-up and the distance of a dislocation to the head of the pile-up, respectively. From equations (A 8)-(A 11),

$$
\begin{aligned}
& d_{\alpha}=2 \alpha d_{1 / 2}\left(q^{*}\right), \\
& L_{\alpha}=2 \alpha L_{1 / 2}\left(q^{*}\right) .
\end{aligned}
$$

The properties of parallel pile-ups of screw dislocations are therefore easily deduced from the properties of a pile-up at the centre of the foil as shown by equations (A 9) and (A 10). The results are expressed by equations (19) and (20). The corresponding values of the coefficients $a_{n}, b_{n}, c_{n}, d_{n}$ and $g_{n}$ are given in table 1 for a parallel pile-up of 11 screw dislocations at the centre of the foil $(\alpha=0.5)$.

\subsection{Comparison with a pile-up in an infinite medium}

Let $d_{\infty}$ and $L_{\infty}$ be the values of $d$ and $L$ for a pile-up of $n$ dislocations submitted to an applied stress $-\tau_{\mathrm{a}}$ in an infinite medium. In this case, the force exerted by the 
(a)

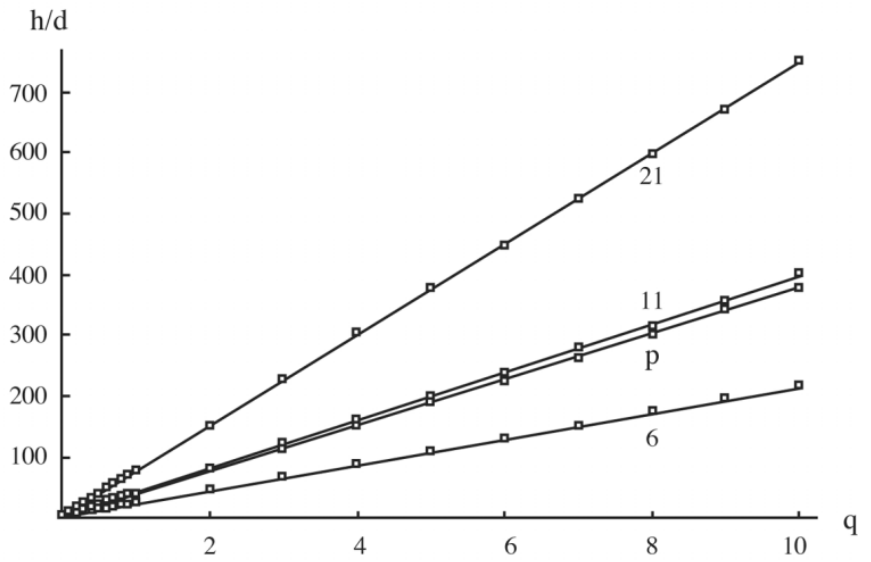

(b) $\mathrm{h} / \mathrm{L}$

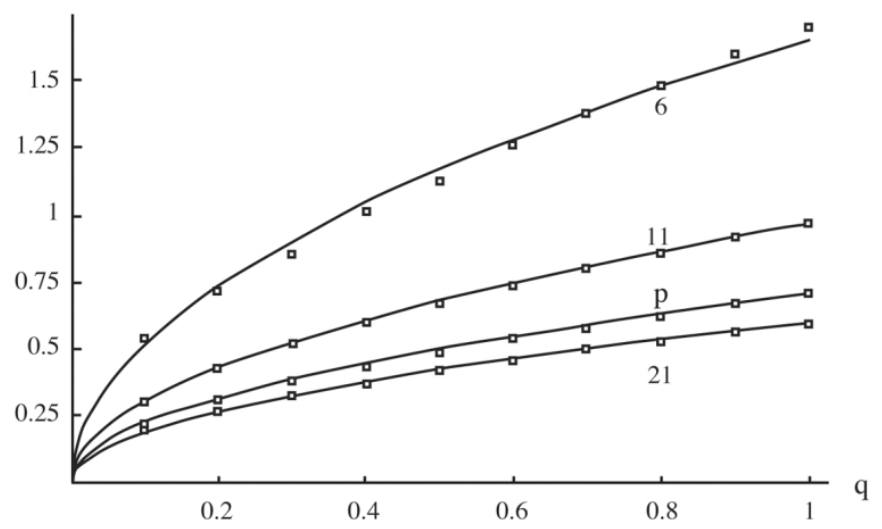

(c)

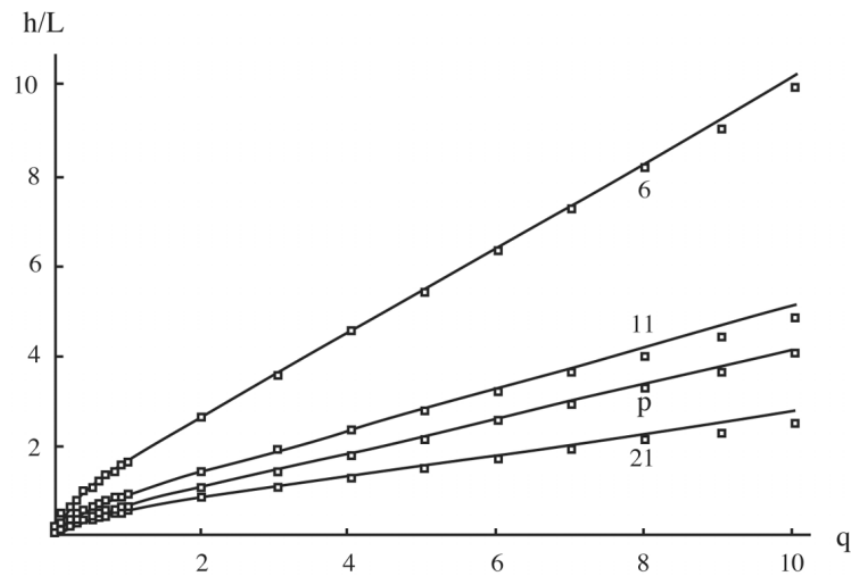

Figure 4. (a) Variation in $h / d$ with $q$ for a pile-up of length $L$ in a thin foil of thickness $h ; d$ is the distance between the first two dislocations. The numbers refer to perpendicular pile-ups of 6,11 and 21 dislocations; $p$ refers to a parallel pile-up with 11 dislocations. (b) Variation in $h / L$ with $q$, for $0.1 \leqslant q \leqslant 1$. (c) Variation in $h / L$ with $q$, for $1 \leqslant q \leqslant 10$. 
Table 2. Measured positions of the dislocations within the pile-up of figure 5 ( $\gamma \mathrm{MCRe}$ deformed at $\left.25^{\circ} \mathrm{C}\right)$.

\begin{tabular}{lc}
\hline Dislocation & $\begin{array}{c}\text { Measured } \\
\text { position (nm) }\end{array}$ \\
\hline 0 & 0 \\
1 & 31 \\
2 & 81 \\
3 & 177 \\
4 & 294 \\
5 & 405 \\
6 & 582 \\
7 & 770 \\
8 & 1161 \\
\hline
\end{tabular}

screw dislocation $i$ on the parallel screw dislocation $j$ is given by

$$
F_{i j}=\frac{\mu b^{2}}{2 \pi\left(x_{j}-x_{i}\right)} .
$$

The equilibrium positions of the dislocations forming a pile up under stress are given by the zeros of the derivative of the relevant Legendre polynomial (Eshelby et al. 1951). No analytical expression exists for these zeros, but they are tabulated in many textbooks (for example Abramowicz and Stegun (1970)). From the tabulated value, one may write to a very good approximation

$$
\begin{gathered}
d_{\infty} \approx 0.3 \frac{\mu b}{\tau_{\mathrm{a}}}, \\
L_{\infty} \approx k_{n} \frac{\mu b}{\tau_{\mathrm{a}}}, \\
k_{n} \approx 0.3 n-0.7 .
\end{gathered}
$$

Hence, comparison with a perpendicular pile-up in a thin foil gives

$$
\begin{gathered}
\frac{d_{\infty}}{d} \approx 0.3 \frac{a_{n} q+b_{n}}{n q}, \\
\frac{L_{\infty}}{L} \approx k_{n} \frac{c_{n} q+d_{n}}{q}, \quad 1 \leqslant q \leqslant 10, \\
\frac{L_{\infty}}{L} \approx \frac{k_{n} g_{n}}{q^{1 / 2}}, \quad 0.1 \leqslant q \leqslant 10 .
\end{gathered}
$$

Let $\tau_{\mathrm{f}}\left(n, \tau_{\mathrm{a}}, h\right)$ be the stress ahead of a pile-up in the thin foil, and $\tau_{\mathrm{f} \infty}\left(n, \tau_{\mathrm{a}}\right)$ be the stress ahead of a homologous pile-up in an infinite medium. Systematic numerical calculation shows that, to a good approximation,

$$
\tau_{\mathrm{f}} \approx \tau_{\mathrm{f} \infty} \exp \left(-\frac{3 x}{h}\right) .
$$

Here $x$ is the distance from the head of the pile-up. This result is found to be almost independent of $n, \tau_{\mathrm{a}}$ and $h$. It is a consequence of the exponential decay of the stress of one dislocation in a thin foil with distance. 
For a perpendicular pile-up of $n=11$ dislocations stopped at an obstacle we have, using the data in table 1 , for $q=0.2$,

$$
d_{\infty} \approx 1.1 d, \quad L_{\infty} \approx 5.5 L
$$

and, for $q=2$,

$$
d_{\infty} \approx 1.03 d, \quad L_{\infty} \approx 1.9 L
$$

The situation is similar for a parallel pile-up, as can be seen in figure 4 .

In the general case, equations (23) and (25) lead to the following simple conclusions.

(i) The structure of the head of the pile-up as well as the local stress on the first dislocations of the pile-up are not significantly influenced by the thickness of the foil.

(ii) On the contrary, the structure of the pile-up (particularly its length) is strongly modified. The stress field ahead of a pile-up in a thin foil decays very rapidly with increasing distance.

\section{§4. ANALYSIS OF THE PILE-UPS OBSERVED IN THIN FOILS}

Unfortunately, pile-ups of perfect screw dislocations parallel or perpendicular to the foil described above are almost never observed. One rather observes pile-ups inclined with respect to the surfaces, and with a mixed character, similar to that shown in figure 5. Thus, a direct comparison with experiments could lead to faulty

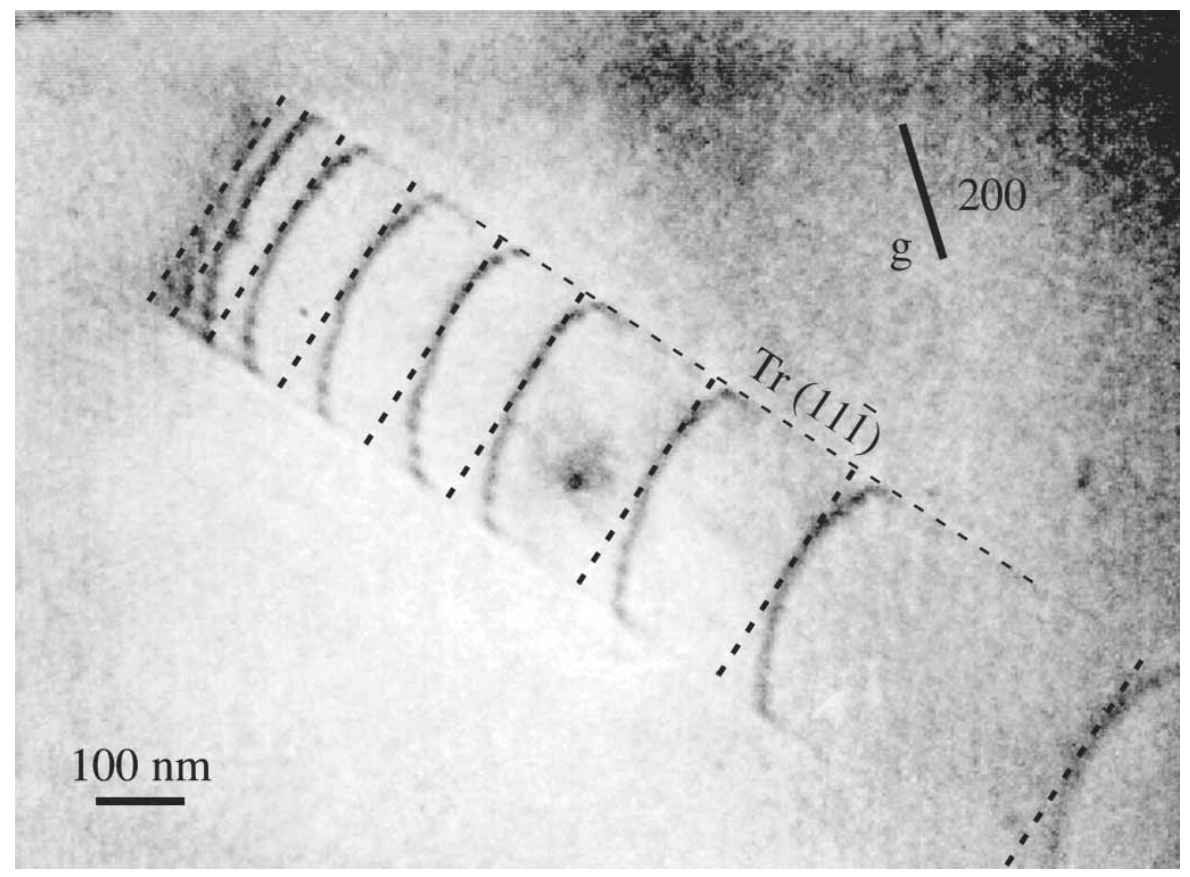

Figure 5. Example of a pile-up found during in-situ deformation of $\gamma_{\mathrm{MCRe}}$ at $25^{\circ} \mathrm{C}$. The dislocations are gliding in the $(11 \overline{1})$ plane. Calculated positions of the dislocations assuming the fault energies reported in figure $6(b)$ are superimposed. 
statements, but it will be shown that, in numerous cases, experimental results can be retrieved by the numerical calculations. The reasons are the following.

(i) Perpendicular and parallel pile-ups give very similar results.

(ii) Equation (6) is obeyed in all cases.

(iii) The most important effect of the stress relaxation at the surface occurs whatever the character and the inclination of the dislocations to the surface. It is well captured by equations (A 4) and (A 7). We may therefore consider that, as is the case in the bulk, these formulae give an acceptable description of the situations, provided that we multiply the functions $\Phi$ and $\psi$ by some constant $\beta$ varying with the character of the dislocations. This does not affect the distribution of the dislocations in the pile-up, although it modifies, by a factor $\beta$, the relation between the applied stress and the length of the pile-up.

(iv) The effect of the relaxation is almost negligible at a distance from the head of the pile-up and small compared with the thickness $h$, where the stress concentration is the largest.

Figure 5 shows an in-situ observation of a pile-up representative of the general situation. Since the dislocations are slightly curved, we have replaced each curved dislocation by an averaged straight dislocation. Knowledge of the position of each dislocation allows the calculation of the elastic interaction forces $R_{p}$ on each dislocation (figure $6(a)$; see also the measured positions of the dislocations in table 2).

Although the existence of a trailing force at the surface is obvious, the observed identical curvatures of all dislocations are directly related to the friction due to the disordered solid solution, which freezes the dislocations in their observed configuration. Precise measurement of the curvature radius of the dislocations shows that the average value of the frictional stress is of the order of $10 \mathrm{MPas}$ (Pettinari-Sturmel et al. 2003). It can therefore be safely neglected in the following discussion.

First of all, it is worth pointing out that the assumption that the order is completely destroyed after the passage of only one or two dislocations is invalidated here since it would impose that $R_{p}$ is constant for $p>2$, which is not the case here nor in previous observations of the same alloy (Pettinari 1999). It must also be emphasized that numerical calculations show that equation (4) is very accurately fulfilled in all cases that have been examined. This indicates that the measured positions of the dislocations are known with good accuracy.

The value of the DAPB energies can then be determined numerically as long as the applied stress $\tau_{\text {eq }}$ at equilibrium is known. This is achieved by using the following assumption: during in-situ experiments, the applied stress on the pile-up is the stress necessary to move the dislocations in the pile-up, and not the stress necessary to create the pile-up. Thus $\tau_{\mathrm{a}}$ must be significantly lower than the elastic limit $\tau_{\mathrm{E}}$. Now let us consider the last dislocation of the pile-up, for which the equilibrium condition is

$$
F_{n}=-b \tau_{\mathrm{eq}}+\left(\gamma_{n}-\gamma_{n-1}\right)+R_{n}+b \tau_{\mathrm{f}}=0 .
$$

As the last dislocation is observed as moving freely in its glide plane, we assume that the order is completely destroyed or at least does not change any longer, that is 
(a)

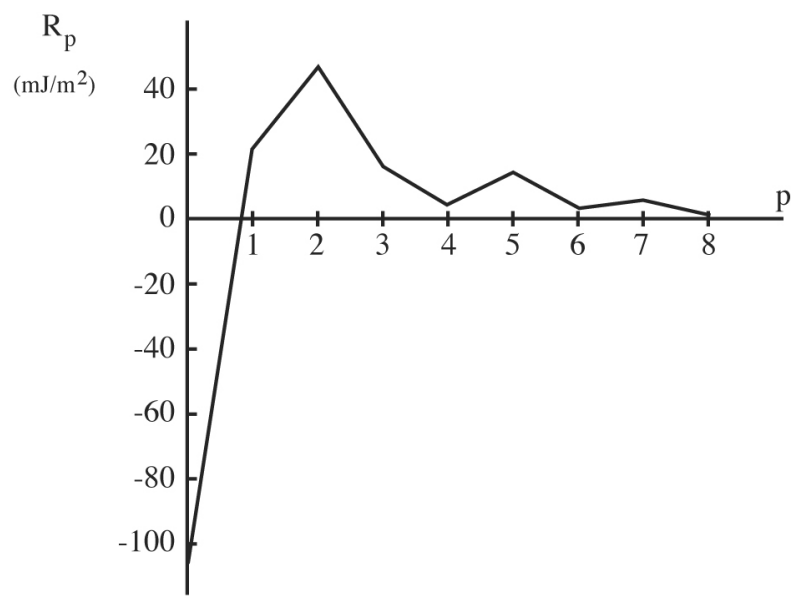

(b)

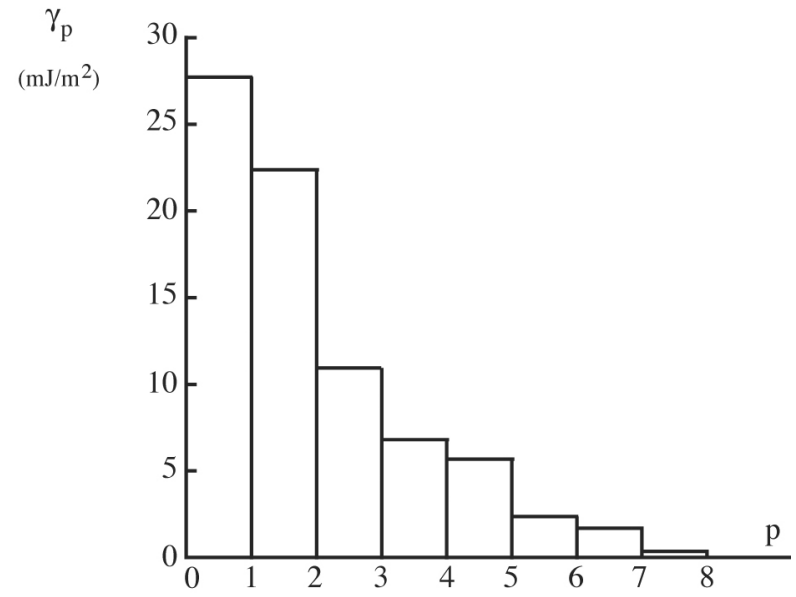

Figure 6. Results of the elastic interaction calculations from the positions of the dislocations in the pile-up in figure 5: (a) elastic interaction force $R_{p}$ as a function of the rank $p$ of the dislocation in the pile-up; (b) energy $\gamma_{p}$ of the defect between dislocations $p$ and $p+1$ resulting from the SRO.

$\gamma_{n}=\gamma_{n-1}\left(n>p^{*}\right)$. Thus, at equilibrium,

$$
b \tau_{\mathrm{eq}}=R_{n}+b \tau_{\mathrm{f}}
$$

Experimentally, it is systematically found that the elastic stress $R_{n}$ on the last dislocation $n$ is small, of the order of or less than a few millijoules per square metre. In consequence, we take at equilibrium for the last dislocation, and thus for all the dislocations,

$$
\tau_{\mathrm{eq}}-\tau_{\mathrm{f}}=0
$$


(a)

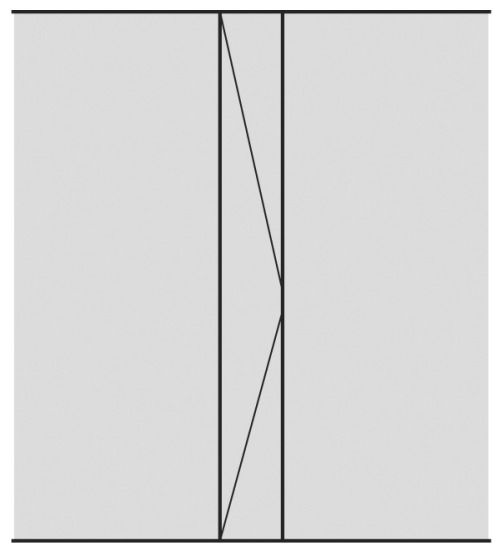

(b)

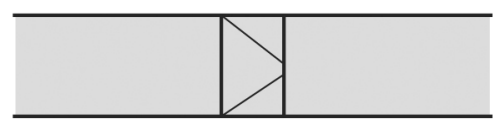

Figure 7. Comparison of the interaction of two parallel dislocations $(a)$ in a thick foil and $(b)$ in a thin foil. As a segment of dislocation interacts with more length of dislocation in the thick foil than in a thin foil, the interaction between dislocations must be significantly higher in the bulk than in a thin foil.

It is worthwhile at this point to discuss results obtained by post mortem observations. We consider a pile-up formed during deformation of the bulk. Then the applied stress is released, the pile-up relaxes but the dislocations of the pile-ups are locked by interaction with impurities, that is by the stress $\tau_{\mathrm{f}}$.

The positions $x_{i}$ of the dislocations in the bulk are given by

$$
\left(\gamma_{p}-\gamma_{p-1}\right)+R_{p}^{(\mathrm{bulk})}\left(x_{i}^{(\mathrm{bulk})}\right)+b \tau_{\mathrm{f}}=0,
$$

where now $R^{\text {(bulk) }}$ is the interaction stress between infinite dislocations. It is easy to show mathematically that the interaction stress between dislocations in the bulk is larger than the interaction stress in a thin foil, as illustrated in figure 7.

If the situation were frozen during the thinning process, the measured positions of the dislocations should agree with the solution of equation (31). However, we consistently found that the measured positions of the dislocations in the pile-ups are characteristic of interactions between dislocations in a thin foil, thus indicating that the dislocations have relaxed after bulk deformation, leading to the idea that the frictional stress has a small influence on the final equilibrium position.

Again, experimentally we systematically found for post mortem observations that the elastic stress $R_{n}$ on the last dislocation $n$ is very small. Thus equation (30) remains valid for post mortem observations as in this case for $p>p^{*}$ :

$$
R_{n}+b \tau_{\mathrm{f}}=0 \quad \text { and } \tau_{\mathrm{a}}=0,
$$

with $R_{n}$ very small. Therefore the frictional stress $\tau_{\mathrm{f}}$ is too small to be measured accurately by this technique.

Equations (10) now simply become

$$
\gamma_{p}=-\sum_{i=0}^{p} R_{i}, \quad 0 \leqslant p \leqslant n .
$$

From the values of the $R_{p}$ the values of the various $\gamma_{p}$ have then been estimated and plotted in figure $6(b)$ for the case of the pile-up depicted in figure 5. Here the 
Table 3. Values of $\gamma_{0}$ and of the frictional stress $\tau_{0}=\gamma_{0} / b$ acting on the first dislocation of a pile-up in the two $\gamma$-phase alloys as functions of temperature $T$.

\begin{tabular}{lrcr}
\hline & $T$ & $\begin{array}{c}\gamma_{0} \\
\left(\mathrm{~mJ} \mathrm{~m}^{-2}\right)\end{array}$ & $\begin{array}{c}\tau_{0} \\
(\mathrm{MPa})\end{array}$ \\
\hline$\gamma \mathrm{MCRe}$ & $\left({ }^{\circ} \mathrm{C}\right)$ & 27.7 & 109.5 \\
$\gamma \mathrm{MCRe}$ & 25 & 33.3 & 131.5 \\
$\gamma \mathrm{MCRe}$ & 350 & 9.8 & 38.9 \\
$\gamma \mathrm{MCRu}$ & 750 & 19 & 75.2 \\
$\gamma \mathrm{MCRu}$ & 25 & 16.7 & 66.1 \\
$\gamma \mathrm{MCRu}$ & 600 & 5.2 & 20.6 \\
\hline
\end{tabular}

effect of the SRO on the motion of dislocations is found to become negligible or small after the passage of about six or seven dislocations. As reported in this figure, the DAPB energies are found to decrease with the passage of the dislocations and to tend to a very small value, if not zero. This strongly suggests the existence of microdomains, of size smaller than $1 \mathrm{~nm}$ (about $4 b$ ), where the occurrence of a $\mathrm{Ni}-\mathrm{Cr}$ pair is favoured in agrement with previous observations (Pettinari et al. 2001).

The residual stress acting on the first dislocation is found to be very high, of the order of $110 \mathrm{MPa}$ for $\gamma$-Re at $25^{\circ} \mathrm{C}$, which corresponds to a fault energy acting against the movement of the first dislocation of the order of $27 \mathrm{~mJ} \mathrm{~m}^{-2}$ and results in a high stress concentration at the head of the pile-up.

Values of $\tau_{0}$ and $\gamma_{0}$, for different temperatures for $\gamma_{\mathrm{MCRe}}$ and $\gamma_{\mathrm{MCRu}}$ are reported in table 3. The decrease in $\gamma_{0}$ with increasing temperature predicted by Schönfeld et al. (1988) and Schwander et al. (1992) is essentially confirmed. Consistently, in the same alloys, $\gamma_{\mathrm{p}}$ decreases with increasing temperature (figure 8).

Finally we note a clear decrease in the $\gamma_{0}-\gamma_{1}$ value at $750^{\circ} \mathrm{C}$, which is in agreement with the vanishing of the pairing of the two first dislocations at the head of the pile-up, as previously reported (Jouiad et al. 1999, Pettinari 1999).

\section{§5. CONCLUSIONS}

(1) We have written the equations of equilibrium of a pile-up of dislocations in a short-range-ordered alloy, under the effect of an applied stress, taking into account both the frictional stress due to local interaction with foreign atoms and the frictional stress resulting from SRO. We have taken into account that the SRO may be modified in different ways by the successive dislocations and shown that the analysis of the distribution of the dislocations in a pile-up provides valuable information on the SRO and its destruction by dislocation glide.

(2) However, such pile-ups can only be observed in thin films. Therefore we have calculated the structure of a pile-up made of screw dislocations in a thin film and shown that it has the following properties.

(i) The stress ahead of the pile-up decreases rapidly with increasing distance.

(ii) The distribution of the first dislocations of the pile-up does not differ very much from that in the bulk.

(iii) The same can be said of the stress on the first dislocation. 
(a)

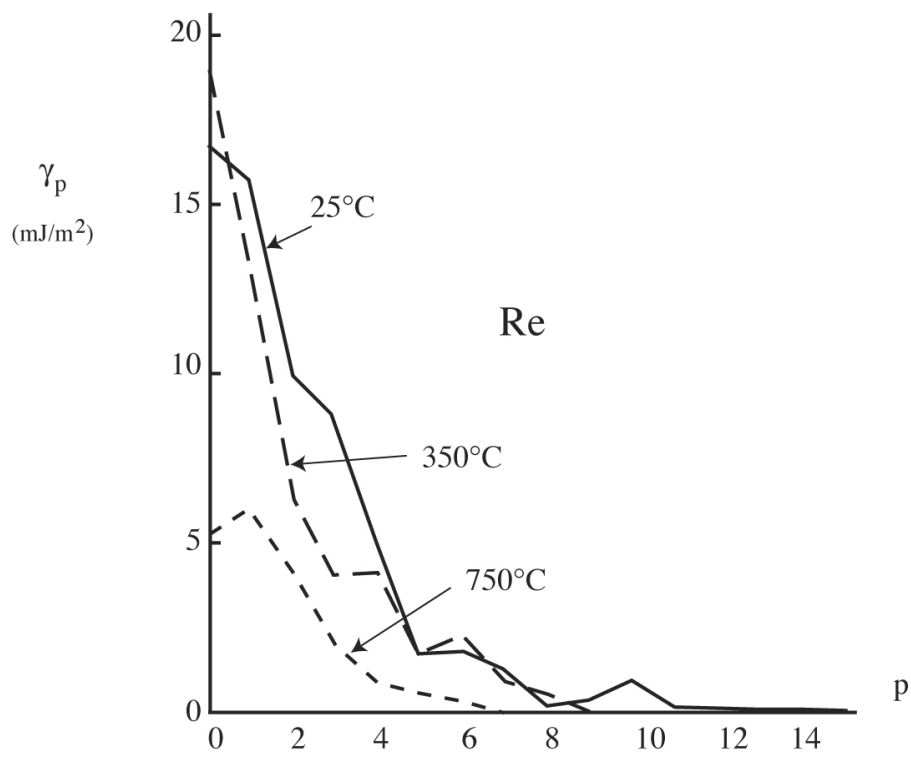

(b)

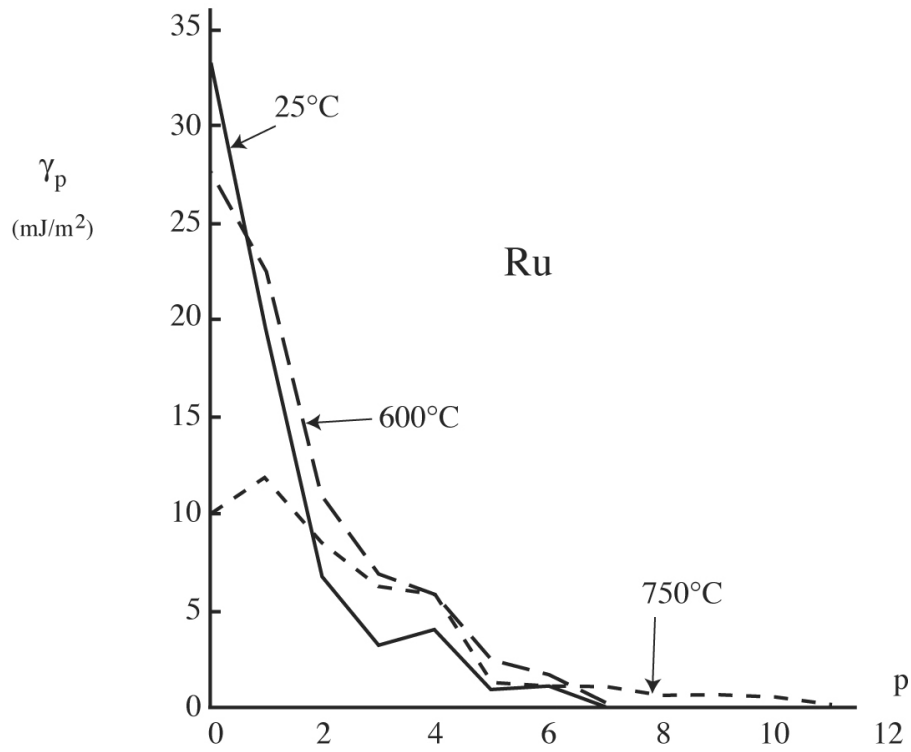

Figure 8. Evolution of the energy $\gamma_{p}$ of the defect created by the successive passage of the dislocations as a function of the deformation temperature (a) for $\gamma_{\mathrm{MCRe}}$ and $(b)$ for $\gamma_{\mathrm{MCRu}}$. Note the clear decrease in the energy with increasing temperature, indicating that the influence of the SRO vanishes with increasing temperature.

(iv) On the contrary, the length of the pile-up as well as the distribution of the dislocations far from the head of the pile-up are strongly modified.

(v) These features may be safely assumed to be general. Analysis of distribution of dislocations in both post mortem and in-situ deformed samples confirm the necessity of taking the screening effect of the surfaces into account. 


\section{APPENDIX A}

$\S$ A 1. Perpendicular pile-up of screw dislocations in a thin foil

Let us consider a pile up of $n+1$ parallel identical screw dislocations perpendicular to the surface of a foil of thickness $h$ (figure $3(a)$ ), labelled $0,1, \ldots, n$. Let $x_{0}, x_{1}, \ldots, x_{n}$ be their abscissae which are greater than 0 . The dislocation 0 is blocked at the origin $\mathrm{O}$.

Using the variables $u_{i}$ defined by equation (13), the force exerted by the dislocation $i$ on the dislocation $j$ can be written (Eshelby and Stroh 1951)

$$
\begin{gathered}
F_{i j}=\frac{\mu b^{2}}{h} \Phi_{i j}, \\
\Phi_{i j}=\frac{4}{\pi^{2}} \operatorname{sgn}\left(u_{j}-u_{i}\right) \sum_{m=0}^{m=\infty} \frac{1}{2 m+1} K_{1}\left[(2 m+1) \pi\left|u_{j}-u_{i}\right|\right],
\end{gathered}
$$

where $K_{1}$ is the modified Bessel function of the first order. Let $\{u\}$ be the set of the variables $u_{j}$, and define

$$
\Phi_{j}\{u\}=\sum_{i \neq j} \Phi_{i j} .
$$

The force $F_{j}$ per unit length exerted on the dislocation $j$ by the dislocations of the pile-up is

$$
R_{j}=\frac{\mu b^{2}}{h} \Phi_{j} .
$$

Assuming all dislocations to be submitted to the same friction force $\tau_{\mathrm{f}}$, and using equations (7) and (14), the total force $F_{j}$ per unit length on the dislocation $j$ is

$$
F_{j}=\frac{\mu b^{2}}{h}\left(\Phi_{j}-q_{j}\right)
$$

At equilibrium all the $F_{j}$ are zero:

$$
\Phi_{j}(\{u\})=q_{j}, \quad j=1,2, \ldots, n+1 .
$$

\section{§ A 2. Parallel pile-up of screw dislocations in a thin foil}

We consider a pile-up of screw dislocations in a plane parallel to the surface, and at a distance $a$ from the closest surface (figure $3(b)$ ). Because of the image force, the stress field of a screw dislocation is that of the dislocation array represented in figure A 1 .

The force $F_{i j / /}$ exerted by the dislocation $i$ on the dislocation $j$ can be written

$$
\begin{gathered}
F_{i j / /}=\frac{\mu b^{2}}{h} \Psi_{i j}, \\
\Psi_{i j}=\frac{1}{4} \frac{\left[1-\cos (2(\pi \alpha)] \sinh \left[\pi\left(u_{j}-u_{i}\right)\right]\right.}{\cosh \left[\pi\left(u_{j}-u_{i}\right)\right]-[1-\cos (2 \pi \alpha)] \cosh \left[\pi\left(u_{j}-u_{i}\right)\right]+\cos (2 \pi \alpha)},
\end{gathered}
$$

where $\alpha=a / h(0 \leqslant \alpha \leqslant 0.5)$. Let

$$
\Psi_{j}(\alpha,\{u\})=\sum_{i \neq j} \Psi_{i j}
$$




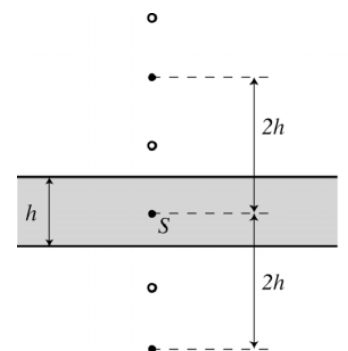

(a)

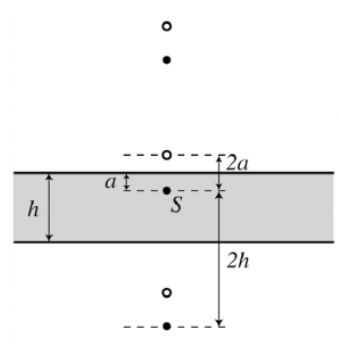

(b)

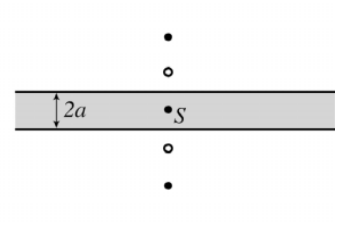

(c)

Figure A 1. Schematic diagram of the stress field of a screw dislocation in a thin foil due to the image force: $(a)$ the dislocation $S$ is at the centre of the foil, and full and open circles represent virtual dislocations with Burgers vectors $+\mathbf{b}$ and $-\mathbf{b}$ respectively; $(b)$ screw dislocation at a distance $a$ from the surface; $(c)$ equivalent configuration of scheme (b) (see text).

The force per unit length exerted by the dislocations of the pile-up on the dislocation $j$ is

$$
R_{j / /}=\frac{\mu b^{2}}{h} \Psi_{j}
$$

The total force on the dislocation $j$ is

$$
F_{j / /}=\frac{\mu b^{2}}{h}\left(\Psi_{j}-q_{j}\right) .
$$

For a pile-up in the centre of the foil, $\alpha=0.5$, equation (A $6 b$ ) can be written

$$
\Psi_{i j}\left(\left(\frac{1}{2}\right), n, u_{j}-u_{i}\right)=\frac{1}{2 \sinh \mathrm{h}\left[\pi\left(u_{j}-u_{i}\right)\right]} .
$$

Numerical calculation shows that, for $\alpha \neq 0.5$,

$$
\begin{aligned}
\Psi_{i j}\left(\alpha, n, u_{j}-u_{i}\right) \approx \Psi_{i j}^{*}\left(\alpha, n, u_{j}-u_{i}\right) & =\frac{1}{4 \alpha \sinh \left[\pi\left(u_{j}-u_{i}\right) / 2 \alpha\right]} \\
& =\frac{1}{2 \alpha} \Psi_{i j}\left(\frac{1}{2}, n, \frac{u_{j}-u_{i}}{2 \alpha}\right) .
\end{aligned}
$$

Let

$$
\Psi_{j}^{*}=\sum_{j \neq i} \Psi_{i j}^{*}
$$

Then

$$
\begin{aligned}
& R_{j / /}=\frac{\mu b^{2}}{h^{*}} \Psi_{j}^{*}, \\
& F_{i j / /}=\frac{\mu b^{2}}{h^{*}} \Psi_{i j}^{*} .
\end{aligned}
$$

The force between two parallel screw dislocations, in a plane $\mathrm{P}$ parallel to the surface of the foil and at a distance $a$ from the closest surface, is to a very good approximation that calculated for screw dislocations at the centre of a foil of thickness $2 a$. 


\section{REFERENCES}

Abramowicz, M., and Stegun, L. A., 1970, Handbook of Mathematical Functions, ninth edition (New York: Dover Publications), p. 771.

Chou, Y. T., Garofalo, F., and Whitmore, R. W., 1960, Acta metall., 8, 480.

Clément, N., 1984, L'Ordre et le Désordre dans les Matériaux, Ecole d'Hiver Aussois, (Paris: Editions de Physique), p. 167.

Clément, N., Coujou, A., Calvayrac, Y., Guillet, F., Blavette, D., and Duval, S., 1996, Microsc., Microanal., Microstruct., 7, 65.

Eshelby, J. D., Frank, F. C., and Nabarro, F. R. N., 1951, Phil. Mag., 42, 351.

Eshelby, J. D., and Stroh, A. N., 1951, Phil. Mag., 42, 1401.

Gerold, V., and Karnthaler, H. P., 1989, Acta metall., 37, 2177.

Jouiad, M., Clément, N., and Coujou, A., 1998, Phil. Mag. A, 77, 689.

Jouiad, M., Pettinari, F., Clément, N., and Coujou, A., 1999, Phil. Mag. A, 79, 2591.

Olfe, J., and Neuhäuser, H., 1988, Phys. Stat. sol. (a), 109, 149.

Pettinari, F., Doctorate Thesis, Toulouse University, France, February 1999.

Pettinari, F., Prem, M., Krexner, G., Caron, P., Coujou, A., Kirchner, H. O. K., and Clément, N., 2001, Acta mater., 49, 2549.

Pettinari-Sturmel, F., Jouiad, M., Kirchner, H. O. K., Clément, N., and Coujou, A., 2002, Phil. Mag. A, 82, 3045.

Pettinari-Sturmel, SaAda, G., Douin, J., Coujou, A., and Clément, N., 2003, Mater. Sci. Engng (submitted).

Prem, M., Krexner, G., Pettinari-Sturmel, F., and Clément, N., 2002, Appl. Phys. A, Suppl., 74, S1112.

Schönfeld, B., Reinhard, L., and Kostorz, G., 1988, Phys. Stat. sol. (b), 147, 457.

Schwander, P., Schönfeld, B., and Kostorz, G., 1992, Phys. Stat. sol. (b), 172, 73. 